\title{
Tendances évolutives des paysages semi-arides anthropisés au Niger: cas des communes rurales d'Aguié, Tchadoua et Gangara (Région de Maradi)
}

\author{
Abdallah MAMADOU BOUREIMA ${ }^{1,2}$, Abdoulaye DIOUF ${ }^{1,2 *}$, \\ Abou-Soufianou SADDA $^{1,2}$, Ali MAHAMANE ${ }^{3}$ et Jan BOGAERT ${ }^{4}$ \\ ${ }^{I}$ Faculté d'Agronomie et des Sciences de l'Environnement, Université Dan Dicko Dankoulodo de Maradi, BP \\ 465 Maradi, Niger. \\ ${ }^{2}$ Unité Mixte de Recherche "Ecologie et Dynamique de la Végétation" (UMR ECODYV), Université Dan Dicko \\ Dankoulodo de Maradi, BP 465 Maradi, Niger. \\ ${ }^{3}$ Faculté des Sciences et Techniques, Université Abdou Moumouni de Niamey, BP 10662 Niamey, Niger. \\ ${ }^{4}$ Unité Biodiversité et Paysage, Gembloux Agro BioTech, Université de Liège, Passage des Déportés, 2. B- \\ 5030 Gembloux, Belgique. \\ *Auteur correspondant ; E-mail: layediouf@yahoo.fr
}

\section{RESUME}

Les impacts du changement climatique et des activités humaines constituent les principaux facteurs de modélisation de la structure et composition des paysages au Niger. Cette étude, réalisée dans les paysages anthropisés semi-arides des communes contigües d'Aguié, Tchadoua et Gangara dans la région de Maradi, a pour objectif d'analyser la dynamique spatio-temporelle des paysages, relativement aux changements d'occupation/utilisation des terres. L'utilisation d'une approche cartographique diachronique basée sur le traitement d'images satellitaires Landsat multicapteurs et multidates (MSS 1976, TM 1988 et ETM+ 1999 et OLI/TIRS 2013), a permis de mettre en évidence et de quantifier, pour la période étudiée (1976 à 2013), les quatre principaux processus de transformation spatiale du paysage: l'attrition, l'agrégation, la création et la dissection des tâches. Les résultats révèlent d'une part une extension spatiale des classes « culture pluviale sous parc arboré », « mosaïque plantation/maraîchage » et « habitation », et d'autre part une très forte régression dans les classes « culture pluviale sous parc arboré à arbustif », « formation naturelle » et « mosaïque jachère/pâturage », due essentiellement à la forte anthropisation. En effet, la classe « culture pluviale sous parc arboré » en 2013 couvrait $72,61 \%$ du paysage étudié contre 14,24\% en 1976. La grande pratique de la régénération naturelle assistée (RNA) dans la zone étudiée explique la transformation et la dominance du paysage par les classes cultures sous parc, constituant donc une politique agro-sylvo-pastorale efficace en paysage semi-aride anthropisé.

(C) 2019 International Formulae Group. All rights reserved

Mots clés: Occupation/utilisation des terres, dynamique paysagère, régénération naturelle assistée, impacts anthropiques, Sahel, Niger.

\section{Evolutive trends of semi-arid anthropized landscapes in Niger: case of the rural communes of Aguie, Tchadoua and Gangara (region of Maradi)}

\begin{abstract}
The impacts of climate change and human activities are the main factor for modeling landscapes structure and composition in Niger. This study, carried out in the semi-arid anthropized landscapes of Aguié, Tchadoua and Gangara communes in the region of Maradi, aims to analyse the landscapes spatial-temporal
\end{abstract}


dynamics, relatively to Landuse/Landcover changes. The use of a diachronic cartographic approach based on the processing of multidate and multi-sensor Landsat satellite images (MSS 1976, TM 1988 and ETM + 1999 and OLI / TIRS 2013), allowed to highlight and quantify for the period studied (1976 to 2013 ), the four main processes of landscape spatial transformation: attrition, aggregation, creation and dissection of landscape patches. The results reveal, on one hand, a spatial extension at different levels in the classes "rainfed croplands under tree park", "mosaic plantation / gardening" and "setlements" and on the other hand a very high area regression in the classes "rainfed croplands under tree to shrub park", "natural woody park" and "mosaic fallow / pasture", mainly due to high anthropization. Indeed, the "rainfed croplands under tree park" class covered $72.61 \%$ of the study area in 2013 against $14.24 \%$ in 1976 . The great use of natural regeneration (ANR) technics in the study area explains the transformation and dominance of the landscape by the classes croplands under parks, constituting therefore an effective agro-sylvo-pastoral policy in an anthropized semi-arid landscape.

(C) 2019 International Formulae Group. All rights reserved

Keywords: Landuse/Landcover, landscape dynamics, human impacts, assisted natural regeneration, Sahel, Niger.

\section{INTRODUCTION}

Le changement global, au sens de Bogaert et André (2013) et Bogaert et al. (2014) est l'ensemble des pressions anthropiques et changements climatiques entraînant des modifications de l'occupation du sol et le déclin de la biodiversité. Il se répercute sur la composition et la configuration du paysage (Touré, 2010). Ainsi, au Sahel et au Niger en particulier, la répartition déséquilibrée dans l'espace et dans le temps des précipitations dont découle la récurrence des sécheresses (Ozer et al., 2010) constitue l'une des principales causes de changements d'occupation/utilisation des terres induisant une transformation significative de la structure et de la composition du paysage (Ibro et Assoumane, 2009 ; Viéri et al., 2008 ; Diallo et al., 2011 ; Millogo et al., 2017 ; Abdou et al., 2019). En outre, les importants bouleversements environnementaux observés actuellement résultent également du déboisement incontrôlé et de l'expansion agricole (Ozer et al., 2010). En effet, la forte croissance démographique conduit à une forte demande en terres cultivables d'où une expansion agricole accélérée au détriment des formations naturelles et des aires de pâturage, et une forte saturation foncière (Sadda et al., 2016a). Ceci implique une dégradation des ressources naturelles impactant le fonctionnement des paysages dont dépend la survie des populations locales (Mahamane, 2001; Mama et al., 2013). Cette anthropisation du milieu est en effet reconnue dans plusieurs zones éco-géographiques, comme la principale cause du changement paysager (Diallo, 2011 ; Bamba et al., 2008 ; Barima et al., 2009 ; Mama et al., 2013 ; Bogaert et al., 2014; Mama et al., 2014; Adamou et al., 2015 ; Maâzou et al., 2017). Dans la région de Maradi au Niger par exemple, plusieurs études relatives à la dynamique paysagère ont souligné la forte croissance démographique $(3,4 \%)$ comme force motrice de la dégradation du couvert végétal (Mahamane, 2001 ; Mahamane et al., 2007 ; Sadda et al., 2016b). L'une des conséquences de cette croissance démographique liée à l'exode rurale et le fort taux de natalité est l'expansion des centres urbains engendrant des mutations socioéconomiques, biologiques et environnementales à diverses échelles spatiales (Abdourahamane et al., 2015).

Dans un tel contexte de changement paysager généralisé, une quantification de la dynamique paysagère dans les zones rurales s'avère nécessaire non seulement par rapport aux changements de l'occupation du sol, mais aussi par rapport à la structure du paysage en termes de composition et de configuration spatiale. Ceci nécessite donc l'utilisation d'outils capables de cerner les processus écologiques y afférents à différentes échelles. Parmi ces outils d'analyse de la structure et de la dynamique des phénomènes spatiotemporels, l'exploitation des images satellitaires, couplée aux techniques du système d'information géographique (SIG), est reconnue comme le plus adapté (Bamba et al., 2008 ; Barima et al., 2009 ; Mama et al., 2013 ; Mama et al., 2014), afin de mieux comprendre 
le phénomène de dégradation des paysages naturels, fortement influencés par des pratiques culturales ancestrales et d'aider à la prise de décision pour une gestion durable de ces paysages.

L'objectif global assigné à la présente étude est donc de déterminer la dynamique spatio-temporelle de l'occupation/utilisation des terres dans trois communes rurales contigües (Aguié, Tchadoua et Gangara) au sud-est de la région de Maradi au cours des 38 dernières années (1976 à 2013) à travers une analyse diachronique. Afin d'atteindre cet objectif, trois hypothèses ont été formulées : (i) les changements d'occupation du sol opérés dans la zone d'étude au cours des 38 dernières années (1976-2013) ont modifié la structure et la composition du paysage; (ii) l'analyse diachronique de l'occupation du sol par approche cartographique et application des principes de l'écologie du paysage permettent de bien caractériser les processus de transformation spatiale du paysage des communes d'Aguié, Tchadoua et Gangara; (iii) les transformations paysagères dans la zone d'étude sont fortement influencées par les actions anthropiques.

\section{MATERIEL ET METHODES Zone d'étude}

La présente étude a été conduite dans 3 communes rurales contiguës situées au centresud du Niger entre $7^{\circ} 24^{\prime}$ et $7^{\circ} 56^{\prime}$ de longitude Est et $13^{\circ} 13^{\prime}$ et $13^{\circ} 45^{\prime}$ de latitude Nord. Elles couvrent une superficie de $2800 \mathrm{~km}^{2}$, soit environ 6,7\% de la région de Maradi (Figure 1). Le relief de la zone d'étude se traduit par une organisation toposéquentielle dominée par l'opposition de deux grandes unités géomorphologiques à savoir les vastes plateaux cuirassés à structure interne concentrique et les vallées fossiles très peu digitées. Ces vallées fossiles présentent souvent des axes d'écoulement en tracé de méandres (Figure 1) et des accumulations sableuses à sablo-argileuses massives. Il s'agit de l'unité bas-fond, la plus mise en valeur par les paysans pour les cultures de contre saison. La structure des sols étant généralement liée à la nature du relief, la zone d'étude présente trois types (3) de sols dont les sols dunaires (appelés localement «Jigawa»), les sols ferrugineux tropicaux lessivés (appelés localement «Gueza»), et les sols de bas-fond (appelés localement «Fadama»). Avec une pluviométrie moyenne annuelle de 469,25 mm $\pm 116,02$, calculée sur une période de 32 ans (1983-2014), la zone d'étude se situe en zone de transition soudano-sahélienne, favorable aux activités agropastorales et au maintien d'une couverture végétale assez importante. Elle appartient au compartiment Sud Sahélien Central (B2) dont la végétation est dominée par les fourrés à Combretum micranthum G. Don. sur les plateaux latéritiques, les formations savanicoles sur les terrasses sableuses méridionales et les formations steppiques sur les dunes et dans les vallées sèches (Saâdou, 1990). Les trois (3) communes comptent au total 220 villages administratifs (Yamba, 2004). Avec un taux d'accroissement d'environ 3,4\%, la population est passée de 172960 habitants en 1988 à 276938 habitants en 2001 pour atteindre 492320 habitants en 2012 (INS, 2015). Ce taux d'accroissement, conduit à un doublement de la population tous les vingtdeux (22) ans. L'agriculture essentiellement de type pluvial pratiquée sur les sols dunaires, demeure la principale activité socioéconomique d'une population à majorité rurale. Quant à l'élevage, deuxième principale activité, elle est de type semi intensif et extensif.

\section{Approche cartographique}

Pour l'étude de la dynamique spatiotemporelle du paysage des communes d'Aguié, Tchadoua et Gangara, une approche cartographique basée sur l'utilisation des indices métriques et images satellitaires Landsat a été adoptée. Le choix de ces images repose sur la gratuité et la disponibilité d'une série temporelle de hautes résolutions spectrale (7 à 11 bandes) et spatiale (60 à 30 m) permettant de bien identifier les structures paysagères homogènes (Oszwald et al., 2010). Ainsi, la base de données de l'United States Geological Survey (USGS) a été explorée (le $12 / 01 / 2017$ ), et quatre scènes Landsat à des dates différentes (au pas de 12 ans au moins) ont été sélectionnées et téléchargées : Multi Spectral Scanner (MSS2) du 29/01/1976; Thematic Mapper (TM 4) du 23/02/1988; 
Enhanced Thematic Mapper Plus $($ ETM +7) du 19/10/1999 et Operational Land Imager/Thermal Infrared Sensor (OLI/TIRS du 05/02/2013) du satellite Landsat-8/LDCM (Landsat Data Continuity Mission). Pour faciliter les opérations de traitement et d'interprétation visuelle à l'écran, ces images préalablement orthorectifiées et géoréférencées au système de projection WGS84 (World Geodesic System) / UTM (Universal Transverse Mercator) $32 \mathrm{~N}$, correspondant à la zone d'étude, ont été soumises à des opérations de prétraitement telles que la composition colorée fausse couleur dans l'ordre 4-3-2 pour les MSS 2, TM4 et ETM+7 ; et 5-4-3 pour la OLI/TIRS ; et d'un rehaussement linéaire de contraste à $2 \%$. Afin d'assurer l'homogénéité au cours des opérations de traitements, l'image MSS2 de 1976 a été ré-échantillonnée de 60 à $30 \mathrm{~m}$ de résolution spatiale à l'aide de l'algorithme du plus proche voisin «Nearest Neighbor» qui selon Caloz et al. (1993) conserve les valeurs radiométriques originales de l'image sous le logiciel ENVI 4.5 ®. Pour l'identification des différentes classes d'occupation du sol, la classification supervisée a été utilisée du fait de sa fiabilité à l'aide de l'algorithme " maximum de vraisemblance ». Cet algorithme repose sur des calculs mathématiques assez robustes et fournit des résultats satisfaisants sur la base des caractéristiques statistiques des échantillons représentatifs des classes précédemment identifiées sur l'image prétraitée. Mais pour valider cette classification, 300 points de contrôle préalablement choisis et supposés être représentatifs de chacune des classes d'occupation du sol de l'image classifiée de 2013 ont été introduits dans un GPS et vérifiés leur appartenance sur le terrain. La matrice de confusion, calculée à partir des données de vérité terrain, a permis d'apprécier la fiabilité et la qualité des résultats de la classification à travers des indicateurs de précision: la précision globale, le coefficient Kappa, les erreurs d'excédent et de déficit, la précision de l'utilisateur et la précision du réalisateur. Après validation de la classification, un filtre spatial majoritaire mobile de taille $3 \times 3$ pixels a été appliqué pour éliminer les pixels isolés.
Enfin, les cartes d'occupation du sol ont été réalisées sous le logiciel Arc GIS 9.3@ après vectorisation des cartes.

\section{Analyse de la dynamique paysagère}

Pour quantifier la composition et décrire la structure paysagère aux différentes dates retenues pour la présente étude, plusieurs indices parmi la panoplie qui existe, ont été calculés à l'aide du logiciel Fragstat 4.1. Ainsi, les indices sélectionnés (Tableau 1) pour décrire la composition du paysage à l'échelle des classes sont : (1) le nombre total de taches d'une classe $(n)$ qui détermine son degré d'hétérogénéité ${ }^{\mathrm{et}} / \mathrm{ou}$ fragmentation ; (2) de l'aire moyenne des taches $(\bar{A})$ qui représente le rapport entre la superficie totale des taches d'une classe $(A)$ et le nombre total des taches ( $n$ ) de la classe considérée ; (3) de l'indice de la plus grande tache (ou dominance), qui est la proportion d'aire occupée par la plus grande tache $i$ d'une classe $j\left(\max \left(a_{i j}\right)\right)$ (McGarigal et Marks, 1995) ; (4) de l'indice de diversité de Shannon qui exprime la diversité de taches (Bogaert et Mahamane, 2005). Quant à la configuration au niveau de la classe et du paysage, elle a été analysée à l'aide de : (5) l'indice de contagion qui renseigne sur la texture du paysage à travers une évaluation du degré d'agrégation et de dispersion des taches (Botequilha-Leităo et al., 2006). Sa valeur varie entre 0 et $100(0<$ contagion $\leqq 100)$. Elle est proche de 0 quand les taches de la classe considérée sont équidistantes et désagrégées au maximum, et proche de 100 quand le paysage est composé d'une seule tache de cette classe. Enfin, la mesure du degré d'anthropisation sur la structure spatiale est démontrée par la corrélation inverse de (6) l'indice de la dimension fractale et (7) le logarithme de l'indice de perturbation du paysage $(U)$ (Bogaert et al., 2014 ; Mama et al., 2013). L'indice de la dimension fractale permet d'apprécier l'effet des perturbations extérieures sur un habitat dont ses valeurs varient entre 1 et $2(1 \leqq I F \leqq 2)$. Cet indice est proche de 1 pour les taches de forme régulière ou circulaire et de 2 pour les taches à forme complexe ou irrégulière c'est à dire un éloignement des formes géométriques euclidiennes (Bogaert et al., 2000). Quant à 
l'indice de perturbation du paysage $(U)$, il est défini comme le rapport de l'aire cumulative des classes anthropiques dans le paysage et de l'aire cumulative des classes de formation naturelle, et traduit le niveau d'anthropisation du paysage (Bogaert et al., 2014 ; Mama et al., 2013). L'analyse diachronique, pour identifier les changements d'état d'une classe d'occupation du sol entre deux dates données, a été effectuée en croisant deux à deux les cartes produites sous le logiciel ArcGIS 9.3. Les résultats statiques issus de ce croissement a permis d'élaborer la matrice de transition qui explique clairement en lignes et en colonnes les changements qui se sont opérés au profit ou au détriment de telle ou telle classe au cours de la période considérée; et sur la diagonale, sont exprimées les proportions des classes qui sont restées inchangées au cours du temps (Bamba et al., 2008 ; Mama et al., 2013 ; Mama et al., 2014).

\section{Identification des processus de transformation paysagère}

L'arbre de décision proposé par Bogaert et al. (2004), largement éprouvé pour sa fiabilité (Bamba et al., 2008 ; Barima et al., 2010 ; Mama et al., 2014 ; Abdourahamane et al., 2015; Sadda et al., 2016a) a été utilisé pour détecter et quantifier les processus de transformation paysagère. Il s'agit d'un modèle dichotomique basé essentiellement sur la comparaison des valeurs de trois (3) indices à savoir l'aire $(a)$, le périmètre $(p)$ et le nombre de taches $(n)$ de la classe entre deux dates $t_{0}$ et $t_{1}$ considérées comme données d'entrée pour identifier dix processus de transformation paysagère. Ainsi, lorsque le nombre de taches $n_{1}>n_{0}$ et l'aire $a_{1}<a_{0}$, il devient difficile de déterminer le processus de transformation en question car il existe deux transformations éventuelles : la fragmentation et la dissection. Pour cela, un quatrième paramètre rentre en jeu, le $t_{\text {obs }}$ (ou valeur du rapport de l'aire totale de la classe au $t_{l}$ par rapport au temps $\left.t_{0}: t_{o b s}=\frac{a_{1}}{a_{0}}\right)$. Le seuil retenu par plusieurs auteurs (Barima, 2009 ; Mama et al., 2014 ; Sadda et al., 2016a) à savoir $t=0,5$ a été utilisé pour cette étude. Si $t_{o b s}<t$, il s'agit d'une fragmentation et dans le cas contraire $\left(t_{o b s}>t\right)$, il s'agit d'une dissection.

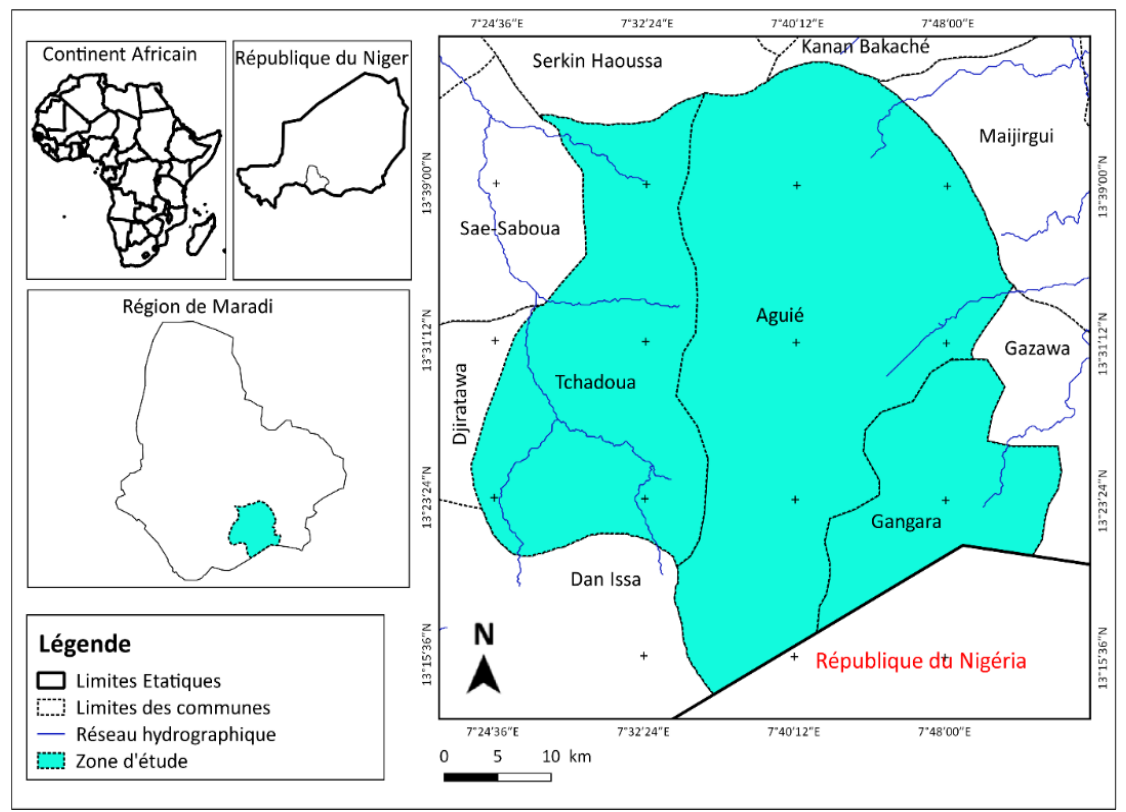

Figure 1: Situation géographique de la zone d'étude. 
Tableau 1 : Indices de structure spatiale.

\begin{tabular}{|c|c|c|c|c|}
\hline INDICES & & FORMULES & & \\
\hline \multirow{4}{*}{$\begin{array}{l}\text { Indices de } \\
\text { composition }\end{array}$} & $\begin{array}{l}\text { Nombre de } \\
\text { taches }\end{array}$ & \multicolumn{3}{|c|}{$\mathrm{N}$} \\
\hline & $\begin{array}{l}\text { Aire moyenne } \\
\text { des taches }\end{array}$ & \multicolumn{3}{|c|}{$\bar{A}=\frac{A}{N}$} \\
\hline & $\begin{array}{l}\text { Indice de } \\
\text { Dominance de } \\
\text { tache }\end{array}$ & \multicolumn{3}{|c|}{$D(\%)=\frac{\max (a i j)}{A} \times 100$} \\
\hline & $\begin{array}{l}\text { Indice de } \\
\text { Diversité de } \\
\text { Shannon }\end{array}$ & \multicolumn{3}{|c|}{$H=-\sum_{i=1}^{N}\left(X_{i} * \ln X_{i}\right)$} \\
\hline \multirow{3}{*}{$\begin{array}{l}\text { Indices de } \\
\text { configuration }\end{array}$} & $\begin{array}{l}\text { Indice de } \\
\text { Contagion }\end{array}$ & Contag $=$ & {$\left[1+\frac{\sum_{i=1}^{N} \sum_{k=1}^{N}\left[\left(P_{i}\right)\left\langle\frac{g_{i k}}{\sum_{k=1}^{N} g_{i k}}\right\rangle\right] *\left[\ln \left(P_{i}\right)\left\langle\frac{g_{i k}}{\sum_{k=1}^{N} g_{i k}}\right\rangle\right]}{2 \ln (N)}\right.$} & $x 100$ \\
\hline & $\begin{array}{l}\text { Dimension } \\
\text { fractale }\end{array}$ & & $D f=\frac{2 \ln \left(0,25 P_{t j}\right)}{\ln a_{t j}}$ & \\
\hline & $\begin{array}{l}\text { Indice de } \\
\text { perturbation du } \\
\text { paysage }\end{array}$ & & $U=\frac{\sum_{\mathrm{i}=1}^{\mathrm{N}} \operatorname{Cia}}{\sum_{\mathrm{i}=1}^{\mathrm{N}} \operatorname{Cin}}$ & \\
\hline
\end{tabular}

Avec $X_{i}$ le rapport de l'aire de la ${ }^{\text {ième }}$ tache de la classe $j$ de celle du total des aires de taches de la classe $j ; P_{t j}$ le total des périmètres de taches de la classe $j$ et $a_{t j}$ le total des aires de taches de la classe $j ; g_{i k}$ est le nombre d'agencement entre les pixels des taches $i$ et $k$ sur la base du double comptage ; $N$ est le nombre de taches et $P_{i}$ le périmètre de la tache $i$; Cia $={ }^{\text {ième }}$ classe anthropique et $\operatorname{Cin}={ }^{\text {ième }}$ classe naturelle.

\section{RESULTATS}

\section{Cartes de l'occupation du sol}

Sept classes d'occupation du sol ont été identifiées (Figure 2): (i) la classe «formation naturelle», constituée des vestiges des peuplements ligneux de Sclerocarya birrea (A. Rich.) Hochst. (Anacardiaceae) dans les forêts classées contiguës de Dan Kada Dodo et Dan Gado en contrat de culture de mil (Pennisetum typhoides (Brum.) Stapf. Hubb.) depuis 2001 (Figure 3, photo 1) et les formations contractées de Guiera senegalensis J.F. Gmel. (Combretaceae) et Combretum micranthum G. Don. (Combretaceae) de la forêt classée de Bakabé (Figure 3, photo 2) ; (ii) la classe «culture pluviale sous parc arboré à arbustif », caractérisée par la dominance de la strate arbustive à Combretaceae de $G$. senegalensis et Combretum glutinosum Perr. ex DC. (Figure 3-photo 3); (iii) de la classe «culture pluviale sous parc arboré», caractérisée par un grand recouvrement des espèces Faidherbia albida (Del.) A. Chev. (Mimosaceae) et Piliostigma reticulatum (DC.) Hochst. (Caesalpiniaceae) (Figure 3, photo 4); (iv) la classe «culture pluviale continue », marquée essentiellement par un recouvrement de champs de mil (Figure 3, photo 5); (v) la classe «mosaïque jachère/pâturage » regroupant les zones de pâturages (Figure 3, photo 6) composés d'un tapis herbacé composé essentiellement de Zornia glochidiata Reich. ex DC. (Papilionaceae); les champs en jachère marqués essentiellement par la dominance 
d'un tapis herbacé à Cassia mimosoides L. (Caesalpiniaceae) et Eragrotis tremula Hochst. ex Steud. (Graminae) (Figure 3, photo 7 ) et les couloirs de passages (Figure 3, photo 8) composés essentiellement de Sida cordifolia L. (Malvaceae) ; (vi) la classe «mosaïque plantation/maraîchage» qui représente surtout la vallée à Hyphaene thebaica (L.) Mart. (Arecaceae) (Figure 3, photo 9) caractérisée par la pratique des cultures maraîchères et des plantations ; et (vii) la classe «habitation» qui présente les habitations (Figure 3, photo 10). Les précisions globales pour les quatre classifications effectuées varient entre $78 \%$ à $97,15 \%$ et l'indice Kappa de $72 \%$ à $96 \%$.

\section{Evolution de la structure du paysage}

Sur la période d'étude (1976 - 2013), les différentes classes d'occupation du sol se caractérisent par une évolution spatiotemporelle en termes de composition et de configuration, à tendance régressive et/ou progressive (Figures 4 et 5) selon les classes et les périodes intermédiaires $(\mathrm{T} 1=1976$ $1988 ; \mathrm{T} 2=1988-1999 ; \mathrm{T} 3=1999-2013 ;$ et $\mathrm{T}$ ou Période globale $=1976-2013$ ) considérées. Quant aux matrices de transition des périodes intermédiaires, elles ont traduit les provenances spatiales des différentes tendances régressives et/ou progressives entre les classes (Tableau 2). Toutefois, celle de la période globale de 1976 à 2013 a permis de mieux interpréter la variation de superficie totale de chacune des classes. Ainsi, la classe « Culture pluviale sous parc arboré à arbustif » a connu la plus forte régression spatiale avec $-50,03 \%$ de conversion (soit plus de la moitié de la superficie totale de la zone d'étude) contre seulement $5,66 \%$ de progression. Par conséquent, elle constituait la principale pourvoyeuse d'espace. En effet, durant la période globale de l'étude, les aires totales et indices de dominance des classes «Habitation», «Culture pluviale sous parc arboré» et «Mosaïque plantation/maraîchage» ont connu une augmentation significative continue, contrairement à ceux des classes «Culture pluviale sous parc arboré à arbustive»
«Formations naturelles» et «Mosaïque jachère/pâturage » qui ont fortement diminué (Figure 4A). Toutefois, pour la «Mosaïque jachère/pâturage» on dénote une forte augmentation de l'indice de dominance au cours de la T1. Pour la classe «Culture pluviale continue » l'indice de dominance et l'aire totale, après une augmentation au cours de $\mathrm{T} 1$, ont connu une diminution progressive. Par contre, l'aire totale de la classe «Culture sous parc arboré à arbustif», matrice du paysage en $\mathrm{T} 1$, a fortement diminué aux cours des différentes périodes intermédiaires essentiellement au profil de la classe «Culture pluviale sous parc arboré ». Cette dernière, à partir du T2 est devenue la matrice du paysage. Quant à l'aire moyenne de la tâche des classes, sa valeur a connu une hausse significative continue («Habitation» et «Formations naturelles ») ou en dents de scie («Mosaïque plantation/maraîchage») (Figure 4B). En effet, au cours de T1, l'aire de la tache moyenne a diminué au niveau des classes relatives aux cultures pluviales et celle de "Mosaïque jachère/pâturage», avant de connaître une forte augmentation dans celles de cultures pluviales à partir de $\mathrm{T} 2$. Une baisse du nombre de taches d'une classe suggère que la classe devient de moins en moins hétérogène ou fragmentée tandis que la diminution de la valeur de l'indice de dimension fractale révèle le passage d'une forme complexe des taches à une forme de plus en plus régulière ou circulaire. Ainsi, au cours de la période globale, les valeurs de l'indice de dimension fractale (Figure 5B) et de celles du nombre de taches (Figure 5A) de toutes les classes liées aux activités humaines hormis la classe «habitation » ont, de manière générale, diminué, avec toutefois une tendance régressive en forme de cloche ou en dents de scie. En effet, les valeurs de ce dernier ont d'abord fortement augmenté au cours de T1, pour la plupart de ces classes, ou en $\mathrm{T} 1$ et $\mathrm{T} 2$ pour les classes «Culture pluviale continue» et «Mosaïque plantation/maraîchage ». Quant à la classe «Formation naturelle», le nombre de taches a diminué de manière progressive durant la période globale. Seuls les indices de la classe 
«Habitation» sont caractérisés par une tendance progressive continue. $\mathrm{La}$ forte corrélation négative observée entre l'indice de dimension fractale et l'indice de perturbation révèle un degré élevé d'anthropisation du paysage étudié (Figure 5B), dont parmi les conséquences une régression significative de la superficie totale des formations naturelles et de la diversité des taches du paysage durant $\mathrm{T}$ (respectivement Figure 4A et 5B), et une diminution de la complexité de forme de leurs taches. En effet, les grosses taches de la classe «Formation naturelle », avec une valeur élevée d'indice de dimension fractale, due à leurs formes naturelles plus complexes en 1976 sont progressivement remplacées par de taches plus petites aux formes de plus en plus régulières (Figure 2) et donc à valeur de $D f$ plus faible. Par ailleurs, à faible valeur d'indice de contagion en 1976 (Figure 5B), la texture du paysage de la zone d'étude se caractérise par des taches équidistantes à désagrégation maximale. Cependant, l'évolution antagoniste de la valeur de cet indice à partir de 1988 (de plus en plus proche de 100) et de l'indice de diversité des taches, montre une tendance de plus en plus dominée par une seule tache, celle de la classe «Culture pluviale sous parc arboré » (Figure 4A) marquée par une baisse de diversité des taches du paysage (Figure 5B).

\section{Processus de transformation paysagère}

Sur la base de l'arbre de décision proposé par Bogaert et al. (2004), divers processus de transformation ayant influencé la dynamique spatio-temporelle du paysage de la zone d'étude ont été mis en évidence. Ainsi, quatre processus de transformations spatiales ont marqué le paysage de la zone étudiée à savoir l'attrition $\left(n_{1}<n_{0}\right.$ et $\left.a_{1}<a_{0}\right)$; la création $\left(n_{1}>n_{0}\right.$ et $\left.a_{1}>a_{0}\right)$; la dissection $\left(n_{1}>n_{0} ; a_{1}<a_{0}\right.$ et $\left.t_{o b s}>t\right)$; et l'agrégation $\left(n_{1}<n_{0}\right.$ et $\left.a_{1}>a_{0}\right)$. $\mathrm{La}$ fréquence de ces processus de transformation varie selon les périodes intermédiaires. $\mathrm{Au}$ cours de la $\mathrm{T} 1$, le processus d'attrition (ou disparition des taches) a été le principal déterminant des changements au sein des classes «Formation naturelle» et «Mosaïque plantation/maraîchage», tandis que ceux de la classe «Culture pluviale sous parc arboré à arbustif», ont été induits par le processus de dissection des taches. Quant au processus de création des taches, il est à la base des changements structuraux des classes «Culture pluviale sous parc arboré», «Culture pluviale continue», «Mosaïque jachère/pâturage» et «Habitation». En T2, on dénote un processus de création des taches au niveau des classes «Mosaïque plantation/maraîchage» et «Habitation». Par contre, les classes «Formation naturelle», «Culture pluviale sous parc arboré à arbustif» et «Mosä̈que jachère/pâturage» ont subi un processus d'attrition (ou disparition des taches). La classe «Culture pluviale sous arboré», est caractérisée par un processus d'agrégation (ou fusion) des taches. Quant à la classe «Culture pluviale continue», elle a été influencée par le processus de dissection. En T3, le processus d'attrition des taches ou disparition des taches initiales a caractérisé les classes «formation naturelle », «culture pluviale sous parc arboré à arbustif », " culture pluviale continue » et «mosaïque jachère/pâturage ». Par contre, les processus d'agrégation ou fusion des taches ont été plus déterminants au niveau de la dynamique des classes «culture pluviale sous parc arboré », «mosaïque plantation/maraîchage » et «Habitation».

En T, le processus d'attrition a été plus déterminant au niveau de la dynamique des classes "Formation naturelle », "Culture pluviale sous parc arboré à arbustif » et « Mosaïque jachère/pâturage ». Par contre, les classes "Culture pluviale sous parc arboré », «Culture pluviale continue » et « Mosaïque plantation/maraîchage » sont caractérisées par un processus d'agrégation. Enfin la classe «Habitation » est marquée par le processus de création des taches. 


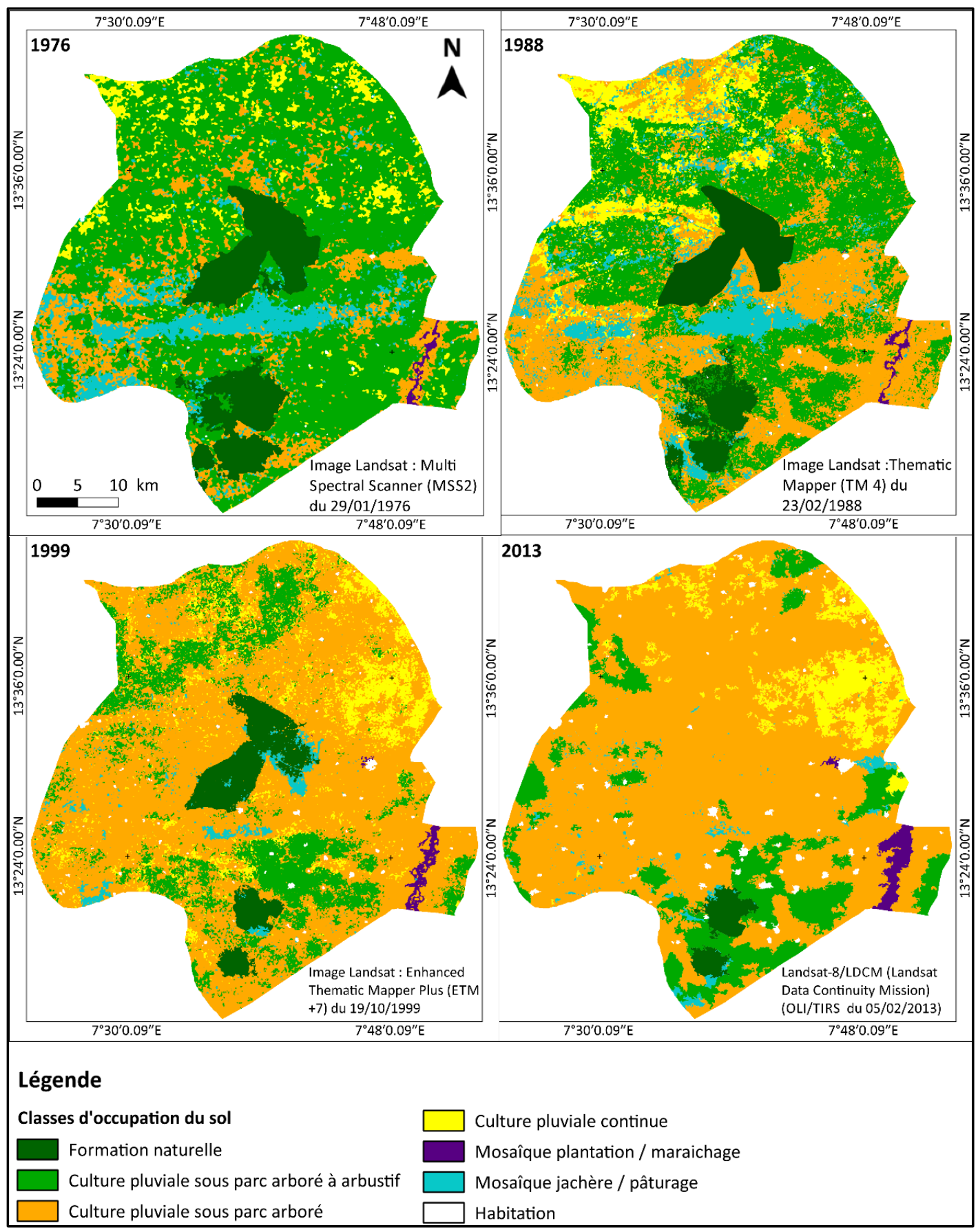

Figure 2 : Etat de l'occupation du sol du paysage des communes d'Aguié, Tchadoua et Gangara en 1976, 1988, 1999 et 2013. 

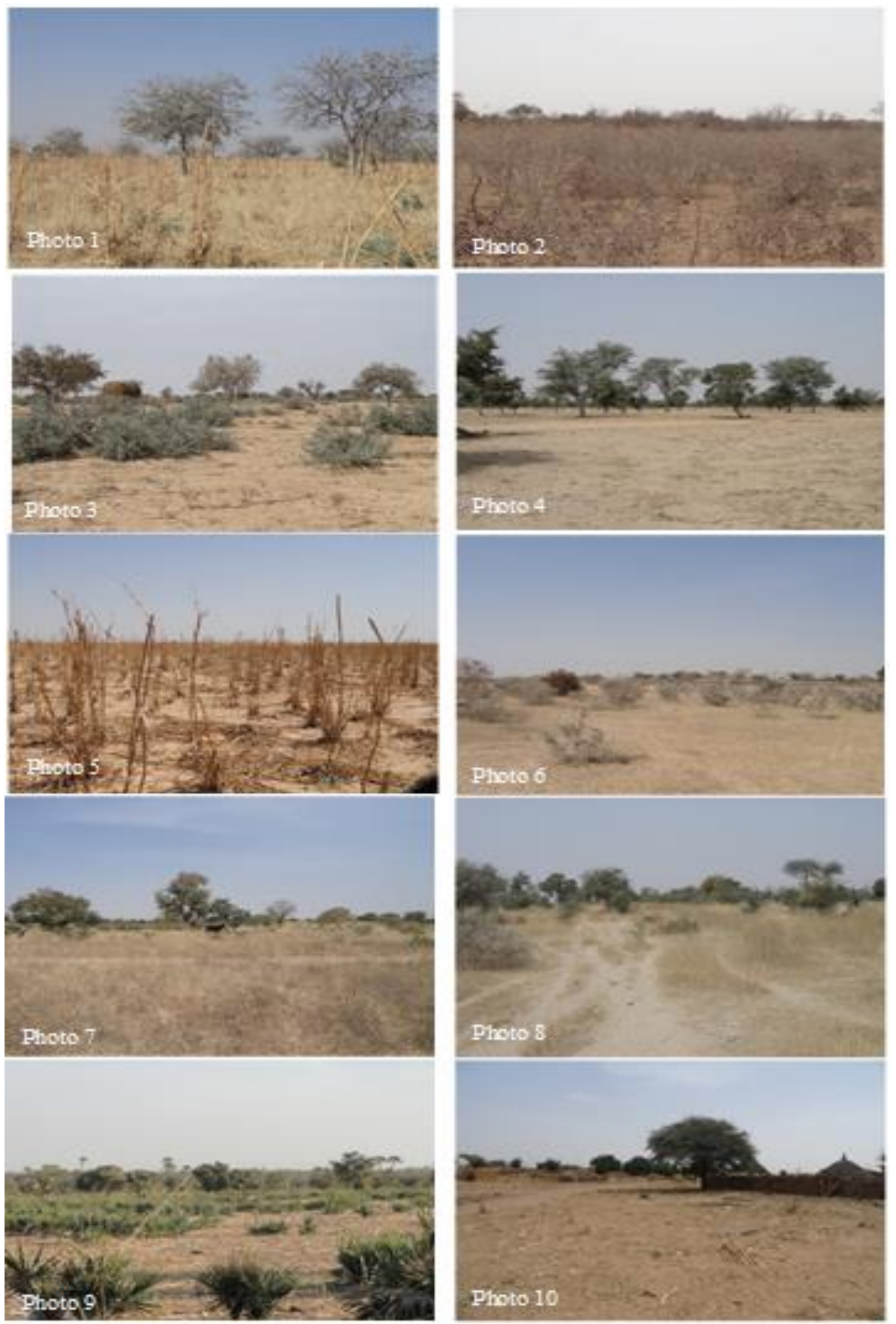

Figure 3 : Classes d'occupation du sol du paysage de la zone d'étude définies. 


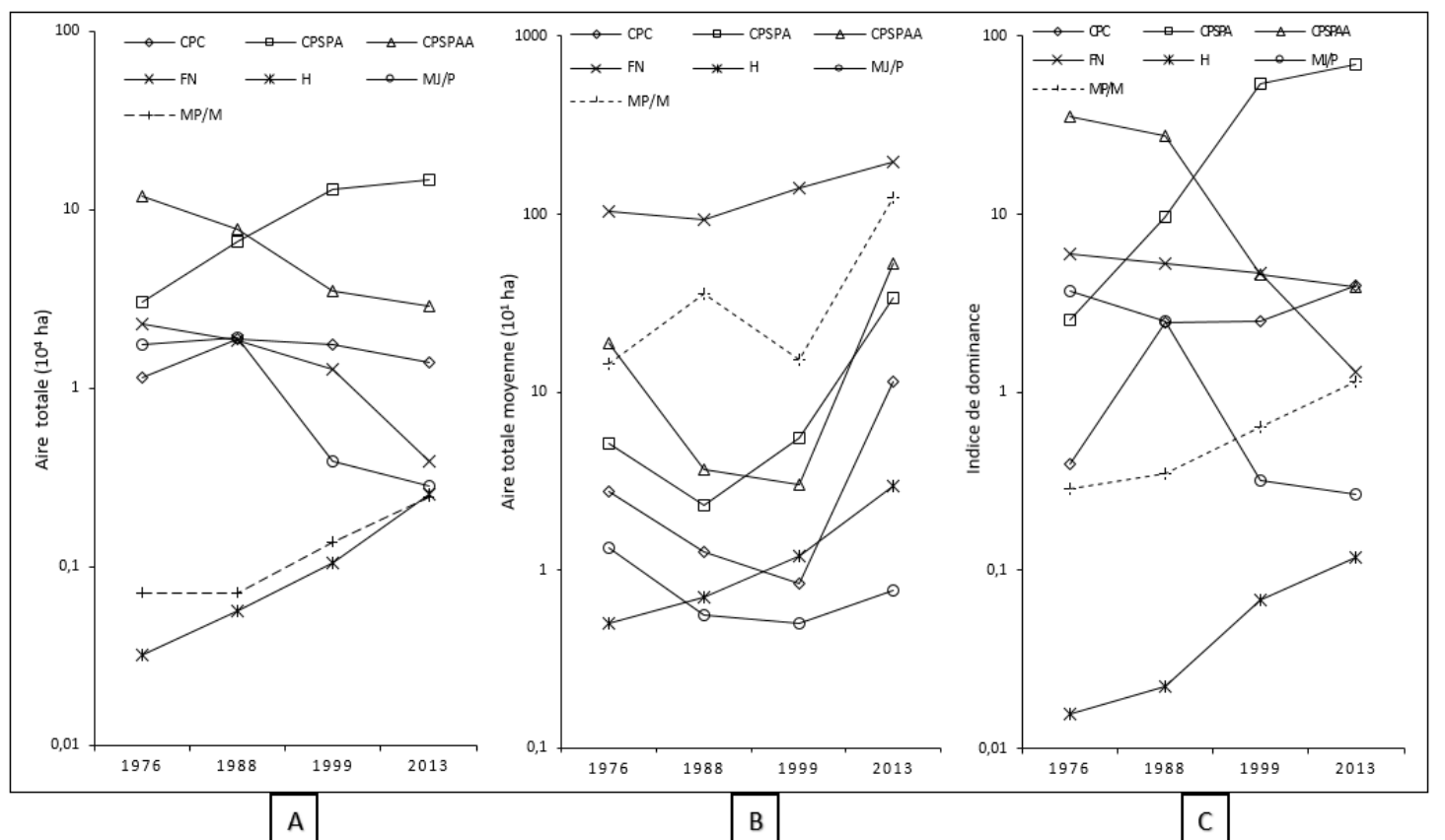

Figure 4 : Evolution de l'aire totale (A), de l'aire moyenne de la tache (B) et de l'indice de dominance (C) des classes d'occupation du sol dans la zone d'étude de 1976-2013.

$F N=$ formation naturelle $; C P S P A A=$ culture pluviale sous parc arboré à arbustive $; C P S P A=$ culture pluviale sous parc arboré; $C P C=$ culture pluviale continue; $M J / P=$ mosaïque jachèrel pâturage; $M P / M=$ mosaïque plantation/maraîchage ; $H=$ Habitation.

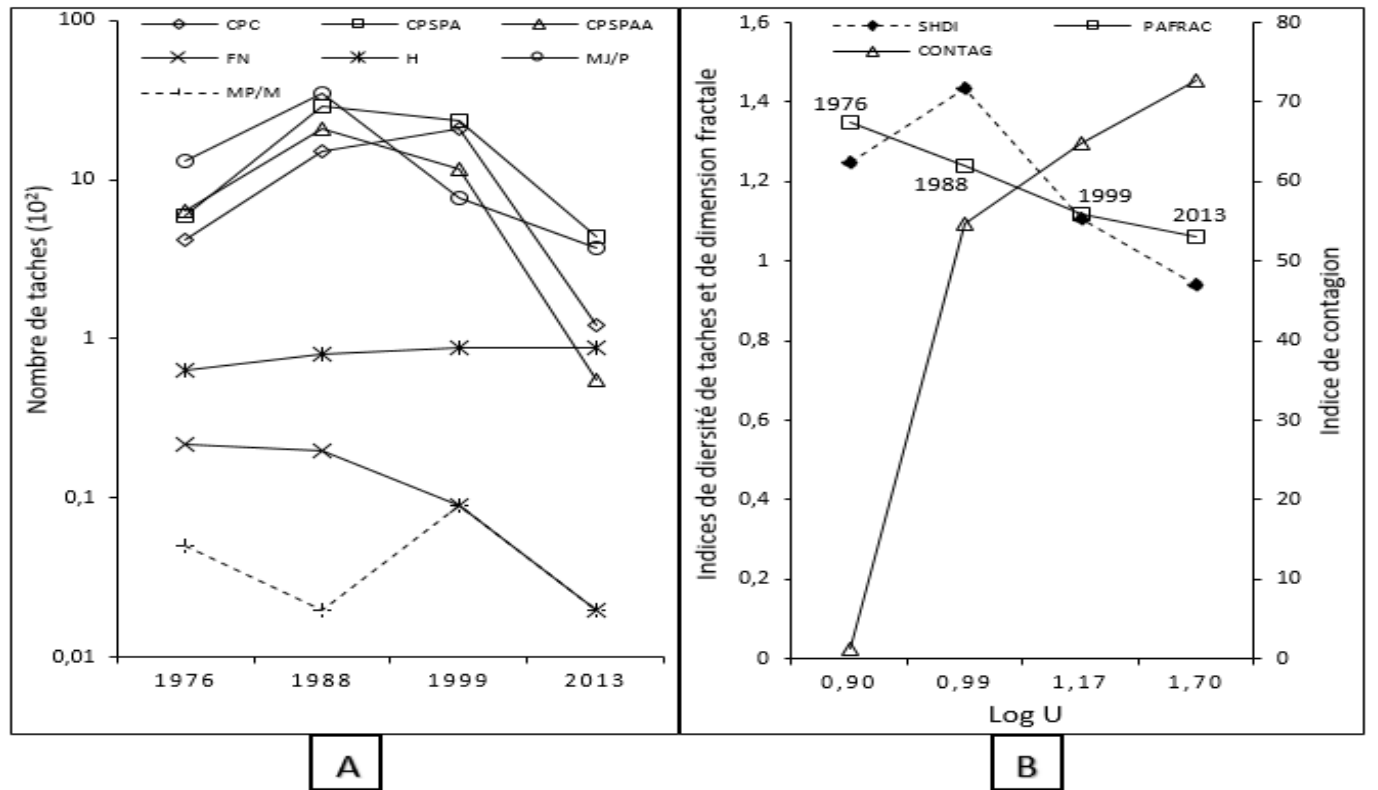

Figure 5 : Evolution du nombre de taches (A), de l'indice de diversité de taches (SHDI ; B), de l'indice de contagion (CONTAG; B) et de l'indice de la dimension Fractale (PAFRAC; B) à l'échelle du paysage de la zone d'étude de 1976-2013.

$F N=$ formation naturelle ; CPSPAA = culture pluviale sous parc arboré à arbustive; CPSPA = culture pluviale sous parc arboré; $C P C=$ culture pluviale continue; $M J / P=$ mosä̈que jachèrel pâturage; $M P / M=$ plantation/maraîchage $; H=$ Habitation. 
Tableau 2 : Matrice de transition de l'occupation du sol (en \%) des communes d'Aguié, Tchadoua et Gangara entre 1976-1988.

\begin{tabular}{|c|c|c|c|c|c|c|c|c|}
\hline \multicolumn{9}{|c|}{ Années 1988} \\
\hline \multicolumn{9}{|c|}{1976} \\
\hline $\operatorname{COS}$ & FN & CPSPAA & CPSPA & CPC & $\mathbf{M J} / \mathbf{P}$ & MP/M & $\mathbf{H}$ & Total \\
\hline FN & 8,30 & 1,72 & 0,67 & 0,04 & 0,51 & 0,00 & 0,00 & 11,24 \\
\hline CPSPAA & 0,38 & 26,46 & 21,16 & 6,68 & 4,13 & 0,01 & 0,10 & 58,92 \\
\hline CPSPA & 0,41 & 5,20 & 6,98 & 0,48 & 1,73 & 0,12 & 0,02 & 14,95 \\
\hline CPC & 0,00 & 2,57 & 0,94 & 1,99 & 0,17 & 0,00 & 0,01 & 5,67 \\
\hline $\mathbf{M J} / \mathbf{P}$ & 0,09 & 2,31 & 2,64 & 0,63 & 2,99 & 0,00 & 0,05 & 8,71 \\
\hline MP/M & 0,00 & 0,00 & 0,13 & 0,00 & 0,00 & 0,21 & 0,00 & 0,35 \\
\hline H & 0,00 & 0,01 & 0,03 & 0,00 & 0,00 & 0,00 & 0,10 & 0,16 \\
\hline Total & 9,19 & 38,27 & 33,03 & 9,34 & 9,54 & 0,35 & 0,28 & 100 \\
\hline \multicolumn{9}{|c|}{1999} \\
\hline \multicolumn{9}{|l|}{1988} \\
\hline $\operatorname{COS}$ & FN & CPSPAA & CPSPA & $\mathrm{CPC}$ & $\mathbf{M J} / \mathbf{P}$ & MP/M & $\mathbf{H}$ & Total \\
\hline FN & 5,43 & 0,58 & 2,28 & 0,10 & 0,76 & 0,01 & 0,03 & 9,19 \\
\hline CPSPAA & 0,74 & 9,31 & 23,58 & 4,34 & 0,27 & 0,01 & 0,03 & 38,27 \\
\hline CPSPA & 0,04 & 4,98 & 24,63 & 2,38 & 0,41 & 0,39 & 0,20 & 33,03 \\
\hline CPC & 0,00 & 1,59 & 6,71 & 0,96 & 0,08 & 0,00 & 0,01 & 9,34 \\
\hline $\mathbf{M J} / \mathbf{P}$ & 0,06 & 0,84 & 7,35 & 0,83 & 0,41 & 0,02 & 0,04 & 9,54 \\
\hline MP/M & 0,00 & 0,00 & 0,10 & 0,00 & 0,00 & 0,25 & 0,00 & 0,35 \\
\hline H & 0,00 & 0,00 & 0,05 & 0,00 & 0,00 & 0,00 & 0,22 & 0,28 \\
\hline Total & 6,27 & 17,29 & 64,71 & 8,62 & 1,92 & 0,67 & 0,52 & 100 \\
\hline \multicolumn{9}{|c|}{2013} \\
\hline \multicolumn{9}{|l|}{1999} \\
\hline COS & FN & CPSPAA & CPSPA & CPC & $\mathbf{M J} / \mathbf{P}$ & MP/M & $\mathbf{H}$ & Total \\
\hline $\mathbf{F N}$ & 1,52 & 2,45 & 2,15 & 0,09 & 0,07 & 0,00 & 0,00 & 6,27 \\
\hline CPSPAA & 0,02 & 8,32 & 7,95 & 0,77 & 0,10 & 0,00 & 0,13 & 17,29 \\
\hline CPSPA & 0,29 & 0,10 & 58,96 & 2,94 & 1,14 & 0,58 & 0,69 & 64,71 \\
\hline CPC & 0,00 & 2,85 & 2,60 & 3,13 & 0,03 & 0,00 & 0,00 & 8,62 \\
\hline $\mathbf{M J} / \mathbf{P}$ & 0,11 & 0,57 & 1,13 & 0,00 & $\mathbf{0 , 0 7}$ & 0,01 & 0,02 & 1,92 \\
\hline MP/M & 0,00 & 0,01 & 0,03 & 0,00 & 0,00 & 0,63 & 0,00 & 0,67 \\
\hline $\mathbf{H}$ & 0,00 & 0,04 & 0,05 & 0,00 & 0,00 & 0,00 & 0,42 & 0,52 \\
\hline Total & 1,94 & 14,35 & 72,61 & 6,93 & 1,41 & 1,22 & 1,27 & 100 \\
\hline \multicolumn{9}{|c|}{2016} \\
\hline \multicolumn{9}{|l|}{1976} \\
\hline $\operatorname{COS}$ & FN & CPSPAA & CPSPA & CPC & $\mathbf{M J} / \mathbf{P}$ & $\mathbf{M P} / \mathbf{M}$ & $\mathbf{H}$ & Total \\
\hline FN & 1,86 & 1,68 & 7,23 & 0,13 & 0,31 & 0,00 & 0,03 & 11,24 \\
\hline CPSPAA & 0,02 & 8,69 & 43,50 & 5,47 & 0,32 & 0,16 & 0,76 & 58,92 \\
\hline CPSPA & 0,05 & 2,73 & 10,47 & 0,38 & 0,47 & 0,72 & 0,12 & 14,95 \\
\hline CPC & 0,00 & 0,43 & 4,02 & 1,11 & 0,02 & 0,00 & 0,09 & 5,67 \\
\hline $\mathbf{M J} / \mathbf{P}$ & 0,01 & 0,82 & 7,35 & 0,10 & 0,29 & 0,00 & 0,14 & 8,71 \\
\hline MP/M & 0,00 & 0,00 & 0,01 & 0,00 & 0,00 & 0,34 & 0,00 & 0,35 \\
\hline $\mathbf{H}$ & 0,00 & 0,00 & 0,03 & 0,00 & 0,00 & 0,00 & 0,13 & 0,16 \\
\hline Total & 1,94 & 14,35 & 72,61 & 6,93 & 1,41 & 1,22 & 1,27 & 100 \\
\hline
\end{tabular}




\section{DISCUSSION}

\section{Approche méthodologique}

L'utilisation de la haute résolution spatiale des images Landsat $(60 \mathrm{~m}$ et $30 \mathrm{~m})$ des périodes de 1976, 1988, 1999 et 2013 à travers l'approche méthodologique mixte et des indices métriques ont permis une bonne détection et quantification des changements de l'occupation du sol de notre zone d'étude (précision de la délimitation des objets et éléments à la surface de la terre). Ceci corrobore les résultats des études similaires obtenus par Sadda et al. (2016a) dans la commune de Tibiri (région de Maradi) au Niger ; Mama et al. (2013) en paysage de zone soudanienne au nord du Bénin ; Mama et al. (2014) en paysage savane-forêt de la zone soudano-guinéenne au centre du Bénin; Barima et al. (2009) en milieux de transition forêt-savane dans le Département de Tanda (Côte d'ivoire); Bamba et al. (2008) en province du Bas-Congo (R.D. Congo). Toutefois, les plus faibles valeurs d'indices de précision et les plus grandes erreurs d'omission et de commission observées au niveau de la classification de l'image de 1976 s'expliquent par la faible résolution du capteur MSS $(60 \mathrm{~m})$ par rapport aux TM, ETM+ et OLI/TIRS $(30 \mathrm{~m})$. Ainsi, les cartes produites constituent une source d'informations indispensables sur l'occupation du sol de la zone d'étude.

\section{Influence des actions anthropiques sur la dynamique paysagère}

L'effet de l'anthropisation sur la dynamique paysagère se caractérise par une dégradation voire disparition des formations naturelles et une densification de la strate arborée au niveau des zones de culture. En effet, en 38 ans, la matrice du paysage est passée de cultures pluviale sous parc arbustif à arboré à la culture pluviale sous parc arboré. Ce changement pourrait s'expliquer par la mise en œuvre de la politique de Régénération Naturelle Assistée (RNA) à partir de 1983 (Larwanou et Saâdou, 2006). Une vingtaine d'années plus tard, les travaux de Larwanou et Saâdou (2006) et Baggnian et al. (2013) suggéraient une augmentation du nombre des arbres dans les champs suite à la pratique de la RNA et donc une conversion de la classe «Culture pluviale sous parc arboré à arbustif » à la classe «Culture pluviale sous parc arboré ». Le fort taux de croissance $(3,4 \%)$ au sein des trois communes explique l'extension spatiale de la classe «Habitation » et la réduction de la superficie des espaces «Mosaïque jachère/pâturage ». En effet, la régression de ces derniers au profit des champs est la conséquence de l'intensification agricole dans l'optique de répondre à la forte demande en terres de cultures pour nourrir une population à majorité rurale et de forte croissance. D'après Larwanou et al. (2006), la forte pression sur les ressources naturelles a incité la population à une intensification agricole dans les régions de Maradi et Zinder. Selon Sadda et al. (2016b), la forte croissance démographique conduit à une forte saturation foncière à travers une expansion agricole accélérée au détriment des formations naturelles et des «Mosaïque jachère/pâturage ». Quant à l'augmentation des superficies des «Mosaïques plantation/maraîchage », elle pourrait s'expliquer par les programmes de développement mis en place par l'Etat et ses partenaires techniques afin de valoriser les zones humides et par conséquent réduire les crises alimentaires et prévenir la sécheresse.

Dans la zone d'étude, les fortes pressions anthropiques dues à la croissance exponentielle de la population ont significativement influencé la dynamique paysagère. Ceci corrobore les résultats obtenus par Sadda et al. (2016a) dans la commune de Tibiri (région de Maradi) au Niger ; Millogo et al. (2017) dans la localité du Loago de la province du Bam au Burkina Faso ; Mama et al. (2013 et 2014) respectivement en zone soudanienne du nord Bénin et en paysage savane-forêt de la zone soudano-guinéenne du centre Bénin ; Bamba et al. (2008) dans la province du bas Congo (République démocratique de Congo), Barima et al. (2009) en milieux de transition forêtsavane dans le Département de Tanda (Côte d'ivoire) qui montrent la dégradation des paysages naturels et la régression spatiale des 
jachères essentiellement due à une pression démographique de plus en plus croissante. Toutefois, au sahel, la dégradation des paysages naturels est aussi exacerbée par les aléas climatiques (Mahamane, 2001 ; Mahamane et al., 2007 ; Chaïbou et Banoin, 2008 ; Ozer et al., 2010 ; Diallo, et al., 2011 ; Adamou et al., 2015).

\section{Corrélation entre processus de transformation spatiale et indices métriques}

La quantification de la structure spatiale à travers les indices métriques a permis de constater dans chacune des classes d'occupation du sol des communes d'Aguié, Tchadoua et Gangara, une variation de la valeur des indices dans l'espace et dans le temps, mettant ainsi en évidence une dynamique du paysage (Bamba et al., 2008 ; Barima et al., 2009; Mama et al., 2013; Mama et al., 2014). En effet, suivant les périodes intermédiaires, le processus d'attrition conduit à une augmentation de superficie à travers les processus d'agrégation et/ou de création. Ainsi, la création de nouvelles taches s'explique par l'augmentation des vergers agricoles, des mosaïques plantation/maraîchage et des zones habitées sous l'effet de l'urbanisation. Quant à l'agrégation des taches, en revanche, elle s'explique par l'homogénéisation du paysage due surtout à la pratique de la RNA dans les champs. C'est ainsi que les classes «culture pluviale sous parc arboré à arbustif » entre 1976-1988 et «culture pluviale continue» entre 1988-1999 ont connu une dissection $\left(\mathrm{t}_{\mathrm{obs}}\right.$ $>0,5)$.

\section{Conclusion}

La présente étude a permis, grâce à l'application des principes de l'écologie du paysage associées à la télédétection et aux outils du Système d'Information Géographique, de déterminer et quantifier la dynamique paysagère des communes d'Aguié, Tchadoua et Gangara entre les années 1976 et 2013. De façon générale, le paysage de ces communes subit une forte anthropisation entraînant des mutations significatives. Ainsi, la matrice paysagère, représentée auparavant par les cultures pluviale sous parc arboré à arbustif, correspond actuellement aux cultures pluviales sous parc arboré. La grande pratique de la RNA explique la transformation et la dominance du paysage en cultures pluviales sous parc arboré. Quant aux mosaïques jachère/pâturage et formations naturelles, elles ont connu une forte régression voire une tendance à la disparition manifeste. Les processus de transformations spatiales d'attrition, d'agrégation, de création et de dissection des taches ont marqué la dynamique spatiale des différentes classes d'occupations du sol. Ainsi, le processus d'attrition des classes "Culture pluviale sous parc arboré à arbustif», «Formation naturelle» et «Mosaïque jachère/pâturage » est prouvé par à une augmentation de superficie dans les classes «Culture pluviale sous parc arboré», «Habitation» et «Mosaïque plantation/maraîchage» à travers les processus d'agrégation et de création de nouvelles taches.

\section{CONFLIT D'INTERETS}

Les auteurs attestent qu'il n'existe aucun conflit d'intérêts, réel ou perçu.

\section{CONTRIBUTIONS DES AUTEURS}

AMB a collecté et traité les données de terrain, analysé les images Landsat, et rédigé le draft du manuscrit ; $A D$ a fourni les données images et a largement contribué au traitement et analyse des données ainsi qu'à la rédaction et correction du manuscrit; ASS a participé au traitement des images et aux travaux de cartographie; AM et JB ont eu à diriger les travaux de cette recherche et ont corrigé le manuscrit.

\section{REMERCIEMENTS}

Les auteurs remercient la coopération française au Niger pour la bourse d'étude accordée à BOUREIMA MAMADOU Abdallah pour sa formation en Master à l'Université d'Abomey Calavi de Bénin, dans le cadre duquel s'inscrit ce travail. Les auteurs remercient également le Pr SAADOU Mahamane (alors Recteur) pour l'autorisation 
du stage de BOUREIMA MAMADOU Abdallah au Département des Sciences du Sol et de Télédétection (DSST) de l'Université Dan Dicko Dankoulodo de Maradi (UDDM).

\section{REFERENCES}

Abdou IK, Abasse T, Moussa M, Habou R, Soumana I, Bogaert J. 2019. Influence des Pressions Anthropiques sur la Dynamique Paysagère de la Reserve Partielle de Faune de Dosso (Niger). Int. J. Biol. Chem. Sci., 13(2): 1094-1108. DOI:

https://dx.doi.org/10.4314/ijbcs.v13i2.41

Abdourahamane IS, Diouf A, Mamoudou BM, Sadda AS, Mahamane A, Saâdou M. 2015. Dynamics of a third world city: Case of Niamey, Niger. J. Geogr. Reg. Plann., 8(5): 120-130. DOI: https://doi.org/10.5897/JGRP2015.0491

Adamou MM, Alhou B, Nazoumou Y, Alloke G. 2015. Impacts des facteurs climatiques et anthropiques sur les ressources et la qualité des eaux de la mare de Tabalak. Int. J. Biol. Chem. Sci., 9(3): $\quad 1665-1677 . \quad$ DOI: http://dx.doi.org/10.4314/ijbcs.v9i3.4

Baggnian I, Adamou MM, Adam T, Mahamane A. 2013. Impact des modes de gestion de la Régénération Naturelle Assistée des ligneux (RNA) sur la résilience des écosystèmes. $J$. Appl. Biosci., 75: 5742- 5752. DOI: 10.4314/jab.v71i1.98819

Bamba I, Mama A, Neuba DFR, Koffi KJ, Traoré D, Visser M, Sinsin B, Lejoly J, Bogaert J. 2008. Influence des pressions anthropiques sur la dynamique spatiotemporelle de l'occupation du sol dans la province du Bas Congo (République Démocratique du Congo). Sciences \& Nature, 5: 49- 60. DOI: http://dx.doi.org/10.4314/scinat.v5i1.421 51.

Barima Y S S, Barbier N, Bamba I, Traoré D, Lejoly J, Bogaert J. 2009. Dynamique paysagère en milieu de transition forêts savane ivoirienne. Bois For Trop., 299(1): $15-25$. DOI: 10.19182/bft2009.299.a20419
Bogaert J, Ceulemans R, Salvador-Van Eysenrode D. 2004. Decision tree algorithm for detection of spatial processes in landscape transformation. Environ Manage., 33: 62-73. DOI: https://doi.org/10.1007/s00267-0030027-0

Bogaert J, Andre M. 2013. Ecologie du paysage en Afrique subsaharienne. Tropicultura, $\quad 31(1)$ : $\quad 1-2$. http://www.tropicultura.org/content/v31n 1.html

Bogaert J, Vranken I, André M. (2014) Anthropogenic Effects in Landscapes: Historical Context and Spatial Pattern. In: Hong SK, Bogaert J, Min Q. (eds), Biocultural Landscapes. Springer, Dordrecht, p 89-112. DOI: https://doi.org/10.1007/978-94-0178941-7_8

Bogaert J, Rousseau R, Van Hecke P, Impens I. 2000. Alternative area-perimeter ratios for measurement of 2D shape compactness of habitats. Appl Math Comput., 111(1): 71-85. DOI: https://doi.org/10.1016/S00963003(99)00075-2

Bogaert J, Mahamane A. 2005. Ecologie du paysage, cibler la configuration et l'échelle spatiale. Annales des Sciences Agronomiques du Bénin, 7(1): 39-68. DOI:

http://dx.doi.org/10.4314/asab.v7i1.4327 7

Botequilha-Leitao A, Miller J, Ahern J, McGarigal K. 2006. Measuring Landscapes: A Planner's Handbook. Washington, DC: Island Press, p 272. ISBN: 1-55963-899-0.

Caloz R, Collet C. 2001. Création d'une ortho-image à l'aide d'un modèle numérique d'altitude : influence des modes de rééchantillonnage radiométrique. In Dubois J-MM, Cavayas F, Lafrance $\mathrm{P}$ (dir). Télédétection appliquée à la cartographie thématique et topographique. Quatrièmes journées scientifiques du Réseau Télédétection de l'UREF, Montréal, 2123 octobre 1991. Québec, Canada, 
Presses de l'Université du Québec, p 1730.

Chaïbou I, Banoin M. 2008. Analyse diachronique et structurale du paysage agraire de la zone de transition de la réserve transfrontalière de biosphère du $\mathrm{W}$ au Niger. Bulletin de la Recherche Agronomique du Bénin, 62: 34-47. URL: http://www.slire.net/download/730/articl e_4_brab_62_chaibou_et_banoin_analys e_diachronique_et_structurale_du_paysa ge_agraire_de_la_zone_de_transition_de _la_r_serve_...pdf

Diallo H, Bamba I, Barima YSS, Visser M, Ballo A, Mama A, Vranken I, Maiga M, Bogaert J. 2011. Effets combinés du climat et des pressions anthropiques sur la dynamique évolutive de la végétation d'une zone protégée du Mali (Réserve de Fina, Boucle du Baoulé). Sécheresse, 22: 97-107. DOI : $10.1684 / \mathrm{sec} .2011 .0306$

Ibro A, Assoumane G. 2009. Etudes de cas sur l'évaluation de la dégradation des forêts: occupation des sols des forêts classées du Niger et l'analyse des dynamiques de changement, $\mathrm{p}$ 25. www.fao.org/docrep/012/k8268f/k8268f0 O.pdf

INS. 2015. Recensement Général de la Population et de l'Habitat (RGPH) : état et structure de la population du Niger en 2012. Institut Nationale de la Statistiques (INS) / Niger. www.statniger.org > statistique > file > RGPH2O12 , ETAT_STRUCTURE...

Larwanou M, Abdoulaye M, Reij C. 2006. Etude de la régénération naturelle assistée dans la région de Zinder (Niger). IRG, USAID-From the American People/EGAT, $\quad \mathrm{p} \quad 48$. https://www.formadenvironnement.org/RNA_Zinder_usaid.p df frame-niger-study-2006_fr.pdf-

Larwanou M, Saadou M. 2006. Evaluation de la flore et de la végétation dans certains sites traités et non traités des régions de Tahoua, Maradi et Tillabéry. Etude sahélienne, $\quad \mathrm{p} \quad 90$. www.agrhymet.ne/portailCC/images/pdf/ syntheseetudesahel.pdf.
Maâzou R, Rabiou H, Issiaka Y, Abdou L, Saidou SI, Mahamane A. 2017. Influence de l'occupation des terres sur la dynamique des communautés végétales en zone Sahélienne: cas de la commune rurale de Dantchandou (Niger). Int. J. Bio. Chem. Sci., 11(1): 79-92.

DOI: http://dx.doi.org/10.4314/ijbcs.v11i1.7

Mahamane A. 2001. Usages des terres et évolutions végétales dans le département de Maradi. Drylands Research Working Paper Crewkerne, Somerset, RoyaumeUni, p 35. ISSN 1470-9384.

Mahamane A, Saâdou M, Bakasso Y, Abassa I, Ichaou A, Karim S. 2007. Analyse diachronique de l'occupation des terres et caractéristique de la commune de Gobi (Région de Maradi/Niger). Sécheresse, 18(4): 296-304. DOI : 10.1684/sec.2007.0105

Mama A, Sinsin B, De Cannière C, Bogaert J. 2013. Anthropisation et dynamique des paysages en zone soudanienne au nord du Bénin. Tropicultura, 31(1): 78-88. http://www.tropicultura.org/text/v31n1/7 8.pdf

Mama A, Bamba I, Sinsin B, Bogaert J, De Cannière C. 2014. Déforestation, savanisation et développement agricole des paysages de savanes -forets dans la zone soudano- guinéenne du Bénin. Bois For Trop., 322(4): 65-75. DOI: https://doi.org/10.19182/bft2014.322.a31 237

Mcgarigal K, Marks BJ. 1995. Fragstats: Spatial Pattern Analysis Program for Quantifying Structure. Department of Agriculture, Pacific Northwest Research Station General Technical Report PNWGTR-351. Oregon, USA. www.fs.fed.us/pnw/pubs/gtr_351.pdf.

Millogo D, Nikiema AA, Koulibaly B, Zombre NP. 2017. Analyse de l'évolution de l'occupation des terres à partir de photographies aériennes de la localité de Loaga dans la province du Bam, Burkina Faso. Int. J. Bio. Chem. Sci., 11(5): 2133-2143. DOI: https://dx.doi.org/10.4314/ijbcs.v11i5.16 
Oszwald J, Antoine L, Arnauld de Sartre X, Marcello T, Gond V. 2010. Analyse des directions de changement des états de surface végétaux pour renseigner la dynamique $\mathrm{du}$ front pionnier de maçaranduba (para, brésil) entre 1997 et 2006. Revue Télédétection, 9(2): 97-111.

Ozer P, Hountondji Y, Niang A. J, Karimoune S, Laminou Manzo O, Salmon M. 2010. Désertification au sahel : historique et perspectives. Bulletin de la Société Géographique de Liège, 54: 69-84. http://hdl.handle.net/2268/10347

Saadou M. 1990. La végétation des milieux drainés nigériens à l'Est du fleuve Niger. Thèse de Docteur ès Sciences Naturelles, Université de Niamey, p 395.

Sadda AS, Diouf A, Lawali S, Ouedrago M, Bogaert J, Mahamane A. 2016. Pression anthropique et dynamique paysagère en zone rurale semi-aride : Cas de la commune de Tibiri, Région de Maradi (Niger). Tropicultura, $\quad \mathbf{3 4}(2)$. http://www.tropicultura.org/content/v34n 2.html

Sadda AS, Lawali S, Diouf A, Abdourahamane IS, Mahamane A. 2016. Land Crisis and Its Impacts on
Agropastoral Systems in the Commune of Tibiri (Maradi Region)/Niger. Int. J. Pure Appl. Sci., 33(1)-37(2):1-10. http://www.ijopaasat.in/yahoo_site_admi n/assets/docs/1_IJPAST-1042-V33N1V37N2.65110933.pdf.

Toure F. 2010. Contribution de la Télédétection et des SIG dans l'étude de la dynamique des îlots de forêt dense dans la Forêt classée des Monts Kouffé et sa périphérie au Bénin entre (19862006). Mémoire de DESS en Production et gestion de l'information géographique, RECTAS, Campus Universitaire Obafemi Awolowo, Nigéria, p. 69.

Viéri T, Di Vecchia A, Pini G, Laminou AM, Toudjani Z, Maman G. 2008. Approches méthodologiques et outils opérationnels pour la gestion des forêts classées en Afrique de l'ouest : le cas du Niger. Sécheresse, 19(4): 261-267. DOI: $10.1684 / \mathrm{sec} .2008 .0155$

Yamba B. 2004. Les mutations des systèmes agraires et des modes d'usage des ressources naturelles dans la zone centrale du Niger. Revue de Géographie Alpine, 92(1): 97-110. DOI: 10.3406/rga.2004.2282. 